\title{
In silico characterization and Molecular modeling of double-strand break repair protein MRE11 from Phoenix dactylifera $\mathrm{v}$ deglet nour
}

\author{
Imen Rekik ${ }^{1 *}$, Zayneb Chaabene ${ }^{1}$, C. Douglas Grubb², Noureddine Drira ${ }^{1}$, Foued Cheour $^{3}$ and Amine Elleuch ${ }^{1}$
}

\author{
* Correspondence: \\ imenbmc@yahoo.fr \\ 'Laboratory of plant biotechnology, \\ Faculty of sciences of Sfax, \\ University of Sfax, Sfax, Tunisia \\ Full list of author information is \\ available at the end of the article
}

\begin{abstract}
Background: DNA double-strand breaks (DSBs) are highly cytotoxic and mutagenic. MRE11 plays an essential role in repairing DNA by cleaving broken ends through its $3^{\prime}$ to $5^{\prime}$ exonuclease and single-stranded DNA endonuclease activities.

Methods: The present study aimed to in silico characterization and molecular modeling of MRE11 from Phoenix dactylifera $L \mathrm{CV}$ deglet nour (DNMRE11) by various bioinformatic approaches. To identify DnMRE 11 cDNA, assembled contigs from our cDNA libraries were analysed using the Blast2GO2.8 program.

Results: The DnMRE11 protein length was 726 amino acids. The results of HUMMER show that DnMRE 11 is formed by three domains: the $\mathrm{N}$-terminal core domain containing the nuclease and capping domains, the C-terminal half containing the DNA binding and coiled coil region. The structure of DnMRE11 is predicted using the Swiss-Model server, which contains the nuclease and capping domains. The obtained model was verified with the structure validation programs such as ProSA and QMEAN servers for reliability. Ligand binding studies using $\mathrm{COACH}$ indicated the interaction of DnMRE 11 protein with two $\mathrm{Mn}^{2+}$ ions and dAMP. The ConSurf server predicted that residues of the active site and Nbs binding site have high conservation scores between plant species.

Conclusions: A model structure of DnMRE11 was constructed and validated with various bioinformatics programs which suggested the predicted model to be satisfactory. Further validation studies were conducted by $\mathrm{COACH}$ analysis for active site ligand prediction, and revealed the presence of six ligands binding sites and two ligands $\left(2 \mathrm{Mn}^{2+}\right.$ and $\left.\mathrm{dAMP}\right)$.
\end{abstract}

\section{Introduction}

The palm family emerged $\sim 80$ million years ago and represents one of the lineages that radiated early in monocot evolution [1]. The genomic comparative analysis of date palm with other species presents an ideal opportunity to investigate the dynamics of angiosperm gene family evolution. The integrity of the genome is constantly threatened by environmental influences and cellular metabolic processes. DNA double strand breaks (DSBs) are among the most hazardous of all DNA lesions and arise from failures in genome metabolism processes and from exogenous sources. In addition they are important programmed intermediates in DNA metabolism.

(c) 2015 Rekik et al. Open Access This article is distributed under the terms of the Creative Commons Attribution 4.0 International License (http://creativecommons.org/licenses/by/4.0/), which permits unrestricted use, distribution, and reproduction in any medium, provided you give appropriate credit to the original author(s) and the source, provide a link to the Creative Commons license, and indicate if changes were made. The Creative Commons Public Domain Dedication waiver (http://creativecommons.org/ publicdomain/zero/1.0/) applies to the data made available in this article, unless otherwise stated. 
DNA double-strand breaks are highly cytotoxic and mutagenic [2]. DSBs can arise during replication and as products of ionizing radiation and genotoxic chemicals, but are also endonucleolytically generated intermediates in meiosis, mating type switching, and recombination [3]. DSBs are predominantly repaired by two pathways. Nonhomologous end joining directly rejoins DSBs, whereas homologous recombination utilizes a sister chromatid or homologous chromosome as a template for DNA resynthesis and rejoining [4]. The MRE11-Rad50-Nbs1 (MRN) complex is a keystone complex that recognizes double-strand break (DSB) damages and responds with nonhomologous end joining (NHEJ) and homologous recombination (HR) pathways $[5,6]$. In addition to the repair of DNA DSBs and cell cycle checkpoint signaling, the MRN complex plays an important role in telomere maintenance, mating type switching, meiotic recombination, and suppression of gross chromosomal rearrangement [7]. MRE11 plays an essential role in repairing DNA by cleaving broken ends through its $3^{\prime}$ to $5^{\prime}$ exonuclease and single-stranded DNA endonuclease activities, as well as hairpin nuclease activities [8]. In addition, MRE11 provides a surface for other DNA repair proteins and checkpoint factors which link the MRE11 complex activities to a wide variety of cellular processes [9]. Structural studies of archaeal, bacterial and human MRE11 homologs have revealed that MRE11 forms a dimer. These MRE11 homologs consist of the nuclease domain containing the active site and the capping domain, which provides selectivity concerning DNA substrates, and they dimerize through the interaction between the two helices by forming a four helix bundle $[10,11]$. The dimerization of MRE11 is crucial as it functions as a frame for Rad50 and DNA binding [10,11]. Nbs1 (also known as Nibrin or p95) is only present in the eukaryotic MRE11 complex. Nbs1 plays key roles in the DNA-damage checkpoint signaling functions of the MRN complex through interactions with a number of proteins, such as Mdc1 (mediator of the DNA-damage checkpoint 1) and ATM [12]. MRE11 from eukaryotes is formed by two regions: the $\mathrm{N}$-terminal core domain containing the nuclease and capping domains, and the C-terminal half containing the DNA binding and GAR domains $[5,6]$. While the N-terminal domain, which is responsible for Nbs1 binding and nuclease activity, is conserved in all species, the C-terminal domain is distinct only in eukaryote MRE11 [5, 6]. The MRE11 gene has been identified in the genomes of all of the eukaryotes sequenced to date, including the Arabidopsis MRE11 ortholog [13]. The homology between different MRE11 orthologs is the strongest in the $\mathrm{N}$ terminus which contains four conserved phosphoesterase domains, but is less pronounced in the $\mathrm{C}$ terminus of the protein which contains two DNA binding domains [14]. The N-terminal region harbors an Nbs1 interacting domain [15], while at the C-terminal region interacts with Rad50 [8]. Originally, MRE11 was identified in yeast (S. cerevisiae) as a gene required for early steps of meiotic recombination, namely for induction as well as for repair of meiotic DSBs [16]. In this study, we present and analyze for the first time an in-silico characterization and homology modelling of MRE11 from Phoenix dactylifera $\mathrm{v}$ deglet nour (DnMRE11) by various bioinformatic approaches, including motif analysis, secondary structure prediction, 3D structure analysis and phylogenetic tree construction.

\section{Materials and methods}

For homology model prediction of DnMRE11, we have developed a procedure which combined old protocols employed in the previous works. Figure 1 show the overall protocol of DnMRE11 model prediction. 


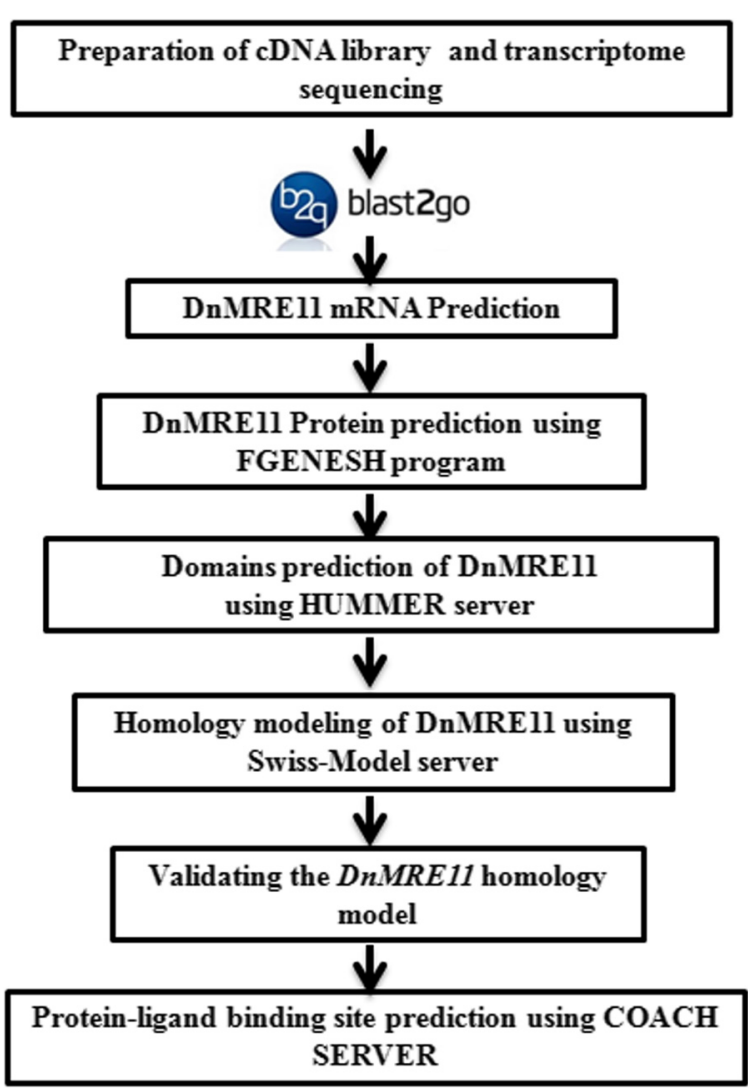

Fig. 1 Overall protocol of DnMRE11 model prediction

\section{RNA preparation}

Total RNA was extracted by modified CTAB method [17] from young leaves of Phoenix dactylifera which were snap-frozen and stored at $-70{ }^{\circ} \mathrm{C}$ until processing. RNA integrity was confirmed using the Agilent 2100 Bioanalyzer with a minimum integrity number value of 8 . Equal amounts of total RNA from each tissue were pooled together for cDNA preparation.

\section{Preparation of cDNA library for transcriptome sequencing}

The poly (A) RNA was isolated from $20 \mu \mathrm{g}$ of the total RNA pool using Dynal oligo (dT) 25 beads (Invitrogen) according to the manufacturer's protocol. Following purification, the mRNA was fragmented into smaller pieces at $70{ }^{\circ} \mathrm{C}$ for $5 \mathrm{~min}$ in the fragmentation buffer (Ambion) and reverse-transcribed to synthesize first strand cDNA using SuperScript III reverse transcriptase (Invitrogen) and N6 random hexamers (Takara). Subsequently, second strand cDNA was synthesized using RNase H (Invitrogen) and DNA polymerase (Invitrogen). These cDNA fragments were further processed by end repair using T4 DNA polymerase, the Klenow fragment of DNA polymerase, and T4 polynucleotide kinase (NEB), and ligation of adaptors with Illumina's adaptor oligo mix and T4 DNA ligase (Invitrogen). The products were gel purified to obtain DNA approximately 200 bp long using Qiaquick Gel Extraction Kit (Qiagen) and enriched with PCR for 
preparing the sequencing library. The quality of the cDNA library was examined by Agilent 2100 Bioanalyzer.

\section{Illumina sequencing}

The cDNA library was sequenced from both of $5^{\prime}$ and $3^{\prime}$ ends on the Illumina GA IIx platform according to the manufacturer's instructions. The conversion of the fluorescent images to sequences, base-calling and quality value calculation were performed by the Illumina data processing pipeline (version 1.4), in which 75 bp paired-end reads were obtained. EST reads obtained from sequencing were cleaned using Seqclean program and assembled by CAP3 [18] using default settings. After assembly, to identified DnMRE11 cDNA, a local BLASTX [19] was used to compare the assembled contigs and singletons against the NR database and analysed using Blast2GO2.8 [20] to provide Gene Ontology, BLAST and domain/Interpro annotation. Candidate mRNA for DnMRE11 from Phoenix dactifera v degelt nour were identified in silico using FGENESH prediction (http://www.softberry.com; with the monocot matrix). Evaluation of $D n M R E 11$ predicted protein was done based on the identification of domains in the NCBI Conserved Domains Database (CDD), phytozome of June 2013 (http:// www.phytozome.net/) and the most recent version of HMMER (HMMERV3.0; [21]).

\section{Sequence alignments}

The DnMRE11 protein sequence was submitted to profile-sequence searches with NCBI, phytozome of June 2013 and most recent version of HMMER. We recovered fifty MRE11 proteins of plants. Protein alignments were performed using MUSCLE [22]. MRE11 proteins were prefixed with the corresponding genus and species initials. Phylogenetic trees were constructed using Phyml software [23] based on the sequence of MRE11 to determine the distribution and evolutionary trend of MRE11 in plants using the Maximum likelihood method with 1000 bootstrapping replicates. The phylogram was generated using EvolView software [24]. After alignement, the fifty MRE11 proteins of plants were submitted to the ConSurf server (http://consurf.tau.ac.il/) for analysis. The ConSurf server assigns relative conservation scores to each residue, taking into account the evolutionary relationships among the family of homologs. The scores are normalized such that the average score is zero, and negative and positive deviations represent the degrees of conservation and variation, respectively. Each residue is then assigned a value 1-9 (1 for most variable, 5 for average, up to 9 for most conserved), which is used for mapping the relative conservation on the molecular surface (see Figure legends). Sequence alignment of DnMRE11, Aeropyrum pernix K1 (AepMRE11, archaea), Homo sapiens (HmsMRE11, animals), Kocuria sp. strain UCDOTCP (KocMRE11, bacteria), Saccharomyces cerevisiae (SacMRE11, fungi) and Galdieria sulphuraria (GasMRE11, protista) was done by ClustalX and viewed with CLC Genomics Workbench (http://www.clcbio.com/).

\section{Homology modeling}

PDB file of DnMRE11 protein was generated by Swiss-Model server (http://www. expasy.org/swissmod/SWISS-MODEL.html). In order to build a model of protein domain, Multiple Sequence Alignment was performed between full length DnMRE11 
protein sequence and another protein domain sequence in this database. To build the model of the DnMRE11 protein with more homology, high resolution (1.80 A) structure of DnMRE11 protein model in Swiss model server was selected as template.

\section{Model reputation}

The backbone conformation of the modeled structure of DnMRE11 with ligand was calculated by analyzing the phi $(\Phi)$ and psi $(\psi)$ torsion angles using Ramachandran plot v 2.0 (http://dicsoft1.physics.iisc.ernet.in/rp/select.html), as determined by Ramachandran plot statistics. The model was further analyzed by, QMEAN [25] and ProSA [26]. ProSA was used for the display of Z-score and energy plots. The volume area dihedral angle for fractional accessible surface area were done with VADAR (http://vadar.wishartlab.com/).

We used $\mathrm{COACH}$ [27] for protein-ligand-binding site prediction for structure-based biological function annotation of DnMRE11. Predicted models were further refined using a side-chain refinement protocol of Discovery Studio 3.5.

\section{Results and discussion}

The MRE11/Rad50 (MR) complex plays a key role in DSB repair. Homologs of MRE11 and Rad50 are found in all kingdoms of life and are essential for genome integrity [28]. After BLAST2GO analyse and searching in annotation results with the keyword "double-strand break repair protein" from the 24,071 transcripts, one MRE11 cDNA was identified (contig 6335). This cDNA has a significant homology with the MRE11 gene of Phoenix dactylifera $\mathrm{v}$ Khalas and other species with more than $90 \%$ sequence similarity (Additional file 1). The results of BLASTX and annotation show that this cDNA is a potential candidate gene of the DnMRE11.

\section{Sequence analysis of DnMRE11 protein}

The DnMRE11 predicted protein length was 726 amino acids. The molecular mass was $81,54 \mathrm{kD}$, and isoelectric point of this protein was 6,25. The predicted localization for the Eukarya domain of DnMRE11 by Predict Protein server was nucleus (GO term ID: GO:0005634). The results of NCBI CDD, pfam and HUMMER analyses show DnMRE11 is formed by three domains: the N-terminal core domain containing the nuclease and capping domains (13 aa, $257 \mathrm{aa}$ ), the C-terminal half containing the DNA binding (302 aa, 456 aa) [5, 6] and a coiled coil region with a hydrophobic surface (Fig. 2a). This specific location on this coiled-coil region interacts with adjacent MRE11 and DNA binding sites on Rad50 and suggests a mechanism for ATP-dependent control of the MRE11 exonuclease by Rad50, by unwinding and/or repositioning DNA ends into the MRE11 active site [5,6]. While the N-terminal domain, which is responsible for Nbs1 binding and nuclease activity, is conserved in all species, the C-terminal (DNA binding and coil) domain is distinct only in eukaryotic MRE11 [5, 6].

\section{Homology model structure analysis of DnMRE11 protein}

The Swiss-Model server was used to predict the 3D sturcture of DnMRE11 based on known crystal structures of homologous proteins (Fig. 2b). The lack of a 3D structure for MRE11 in PDB motivated us to construct the 3D model for MRE11. The most successful techniques for prediction of three dimensional structures of proteins rely on 


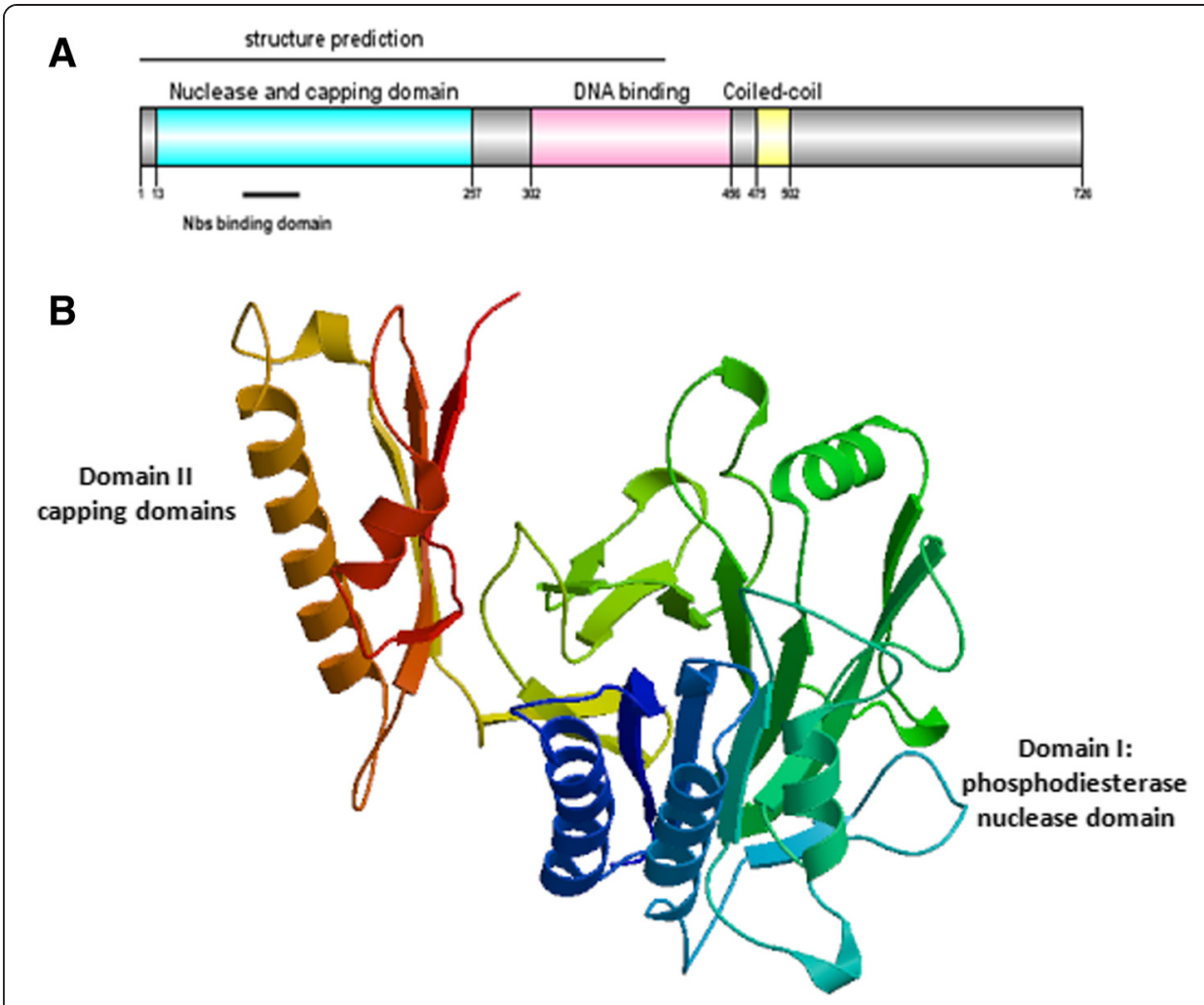

Fig. 2 a Domain features of DnMRE11; (b) 3D structure of DnMRE11

aligning the sequence of a protein of to a homolog of known structure. The highestscoring and validated model for DnMRE11 that exhibits the greatest amino acid sequence identity with the crystal structure is double-strand break repair protein MRE11 of Schizosaccharomyces pombe ScpMRE11 (PDB ID : 4FBK1.A), which is in the MRE11 superfamily. Only 404 residues of the $\mathrm{N}$ terminus (nuclease and capping domains) of DnMRE11 have modelled with $100.0 \%$ confidence by the single highest scoring template (Fig. 2b). This protein is $43.33 \%$ identical to the DnMRE11 protein across $54 \%$ of amino acid sequence. The alignement of two secondary structures of DnMRE11protein and the best template PDB : 4FBK1.A is shown in Additional file 2.

The stereochemical qualities of the predicted models of DnMRE11 proteins were analysed through QMEAN and ProSA servers confirmation was evaluated by the inspection of the Psi/Phi Ramachandran plots.

ProSA was used to check the three- dimensional model of DnMRE11 proteins for potential errors. The program displays 2 characteristics of the input structure: its Z-score and a plot of its residue energies. The ProSA Z-score of -9.5 indicates the overall model quality of DnMRE11 protein (Fig. 3a). Z-score also measures the deviation of total energy of the structure with respect to an energy distribution derived from random conformations. The scores indicate a highly reliable structure and are well within the range of scores typically found for proteins of similar size. The energy plot shows the local model quality by plotting knowledge-based energies as a function of amino acid sequence position (Fig. 3b).

QMEAN analysis was also used to evaluate and validate the model. 


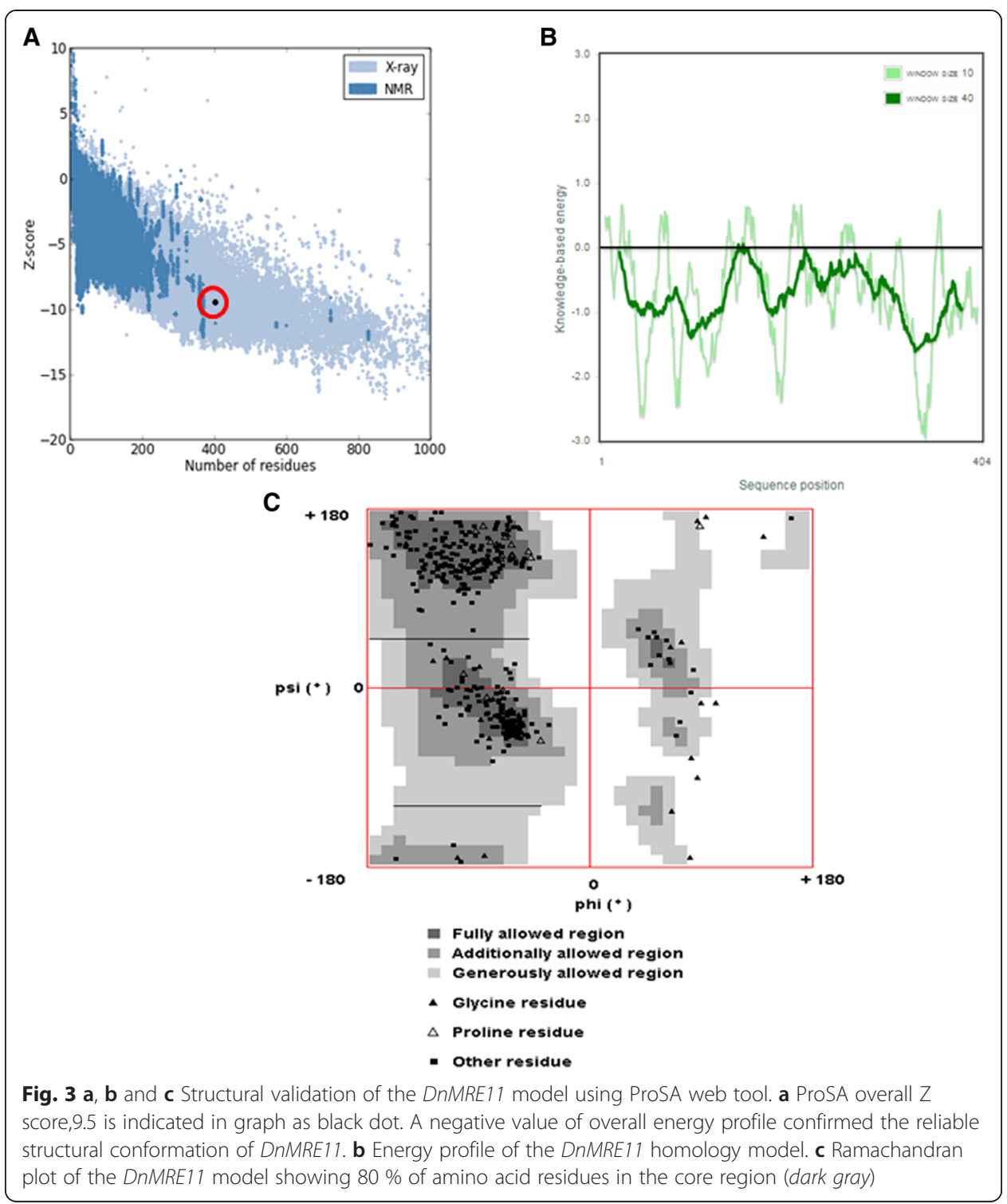

The QMEAN4 score of the model was 0.672 and the Z-score was -1.57 which was close to the value of 0 and this shows the good quality of the model because the estimated reliability of the model was expected to be in between 0 and 1 (Table 1). A comparison between normalized QMEAN score (0.672) and protein size in non-redundant set of PDB structures in the plot revealed different set of Z-values for different parameters such as C-beta interactions (0.38), interactions between all atoms $(-0.23)$, solvation $(-0.49)$ and torsion $(-1.45)$ (Table 1$)$.

The constructed homology model was also evaluated for structural and stereo chemical efficiency. A Ramachandran phi-psi plot for DnMRE11 (Fig. 3c) revealed that $80 \%$ of residues lay in the core region (dark gray), another 15,5\% were in the allowed region (light gray), 2,5\% were in generally region (very light gray) and only $2 \%$ lay in the disallowed region (white). The above analysis of the predicted structure provides solid evidence that the predicted 3D structure of $D n M R E 11$ is of good quality. 
Table 1 Z scores and energy of individual component of QMEAN for DnMRE11 model

\begin{tabular}{llr}
\hline Scoring function term & Energy & Z-score \\
\hline C-beta interactions & -170.63 & 0.38 \\
All-atom pairwise & -10397.83 & -0.23 \\
Solvation & -32.72 & -0.49 \\
Torsion angle & -76.09 & -1.45 \\
QMEAN4 score $=0.672$ & & -1.57 \\
\hline
\end{tabular}

The overall structure of MRE11 proteins of fungi (Schizosaccharomyces pombe) ScpMRE11 (PDB ID:4fbk) [29] and human HmMRE11 (PDB ID: 3t1i) [30], are relatively similar compared with DnMRE11. Both nuclease and capping domain structures are present and the structure of the DnMRE11 nuclease domain is more similar to the equivalent domain from these MRE11 proteins (Fig. 2b) [10, 11].

The DnMRE11 core comprises two $\alpha / \beta$ fold domains, a larger $N$-terminal nuclease domain and a smaller C-terminal capping domain (Fig. 2b, Additionnal file 2). The DnMRE11 nuclease domain, which resembles the calcineurinlike Ser/Thr phosphosesterase, consists of five helices and 13 strands, and the capping domain is composed of three strands packed by two helices on one face (Additionnal file 2).

Domain II, which consists of a three-stranded $\beta$ sheet and two $\alpha$ helices, partially caps the active site phosphodiesterase motifs of Domain I, suggesting that Domain II plays a role in DNA substrate specificity (Fig. 2b). This Domain II cap appears to be a unique MRE11 feature as no equivalent domain or fold is found in the protein phosphatases. In the capping domain of DnMRE11, substantial differences exist in the length and orientations of the loops compared with those of template ScpMRE11. In general, helices and loops in the $D n M R E 11$ capping domain are relatively longer than those of ScpMRE11 (Additionnal file 2, $\beta$ 15).

These results show that the DnMRE11 capping domain with three strands is closer to the canonical structure $[10,11]$.

\section{Active binding site prediction of DnMRE11}

A multiple-sequence alignment revealed that the $D n M R E 11$ protein has low similarity with homologs from others species, such as Aeropyrum pernix K1 (AepMRE11, archaea), Homo sapiens (HmsMRE11, animals), Kocuria sp. strain UCD-OTCP (KocMRE11, bacteria), Saccharomyces cerevisiae (SacMRE11, fungi) and Galdieria sulphuraria (GasMRE11, protista), with the exception of active site residues (Additional file 3). Domain I contains five conserved phosphodiesterase motifs, which form the nuclease active site [30] (Additional file 1). Predection of the active site location of DnMRE11 protein by the DEPTH server (Additional file 4) showed the phosphodiesterase motifs (red color) situated between the nuclease and capping domain. The Domain I fold and active site location resemblethe catalytic domain of calcineurin-like Ser/Thr phosphatases and the DNA base excision repair enzyme apurinic endonuclease 1 (APE1). This resemblance suggests that the di-metal nuclease mechanism of MRE11 is similar to the di-metal protein phosphatase mechanism of Ser/Thr phosphatases [31].

The active site of any protein is critical for its activity, thus blocking it with a suitable ligand may result in inhibition of the protein either partially or completely. In this 
regard, it becomes highly essential to determine the amino acid residues of the protein that forms the active site.

The $\mathrm{COACH}$ server analysis demonstrated that two $\mathrm{Mn} 2+$ ions are coordinated in each of the two apparently functional nuclease sites and the crucial amino acid residues forming the active site of DnMRE11 include ASP20, ASP60, ASN127, HIS225, HIS253 for the one Mn2+ ion (Fig. 4a) with derict interraction with residues ASP60, HIS225 and HIS253 (Fig. 4a). The second Mn2+ interract with ASP20, ASP60, HIS253 and HIS255 (Fig. 4b). The COACH predicts also that the MRE11 active site binds dAMP mainly via the phosphate moiety, which is bound by HIS22, ASP60, GLU286, HIS253, HIS255 and THR280 (Fig. 4c). The double coordination of the dAMP phosphate by both active site metals resembles the binding of phosphorylated protein residues in Ser/Thr phosphatases, further supporting a common phosphoesterase mechanism between MRE11 and Ser/Thr phosphatases [31]. One conserved residue in eukaryotic MRE11 proteins, Glu286 (Fig. 4c), forms H-bonds with HIS253 and stabilizes this histidine. The same active site binding substrates were found using the FunFOLD server [31] and the 3DLigandSite server [32].

Comparing structures of HmsMRE11 (Homo sapiens, PDB : 3T1I) [30], PfMRE11 (Pyrococcus furiosus, archaea PDB ID: 1II7, [33]), Sp MRE11 (Schizosaccharomyces pombe [29], PDB : 4fbkA) and DnMRE11 protein, we conclude that the residues which bind the metals and dAMP are the same at the active site for all these species. While there are overall similarities between the active site of DnMRE11, bacterial and archaeal MRE11 proteins, some differences are observed. These differences are largely limited to

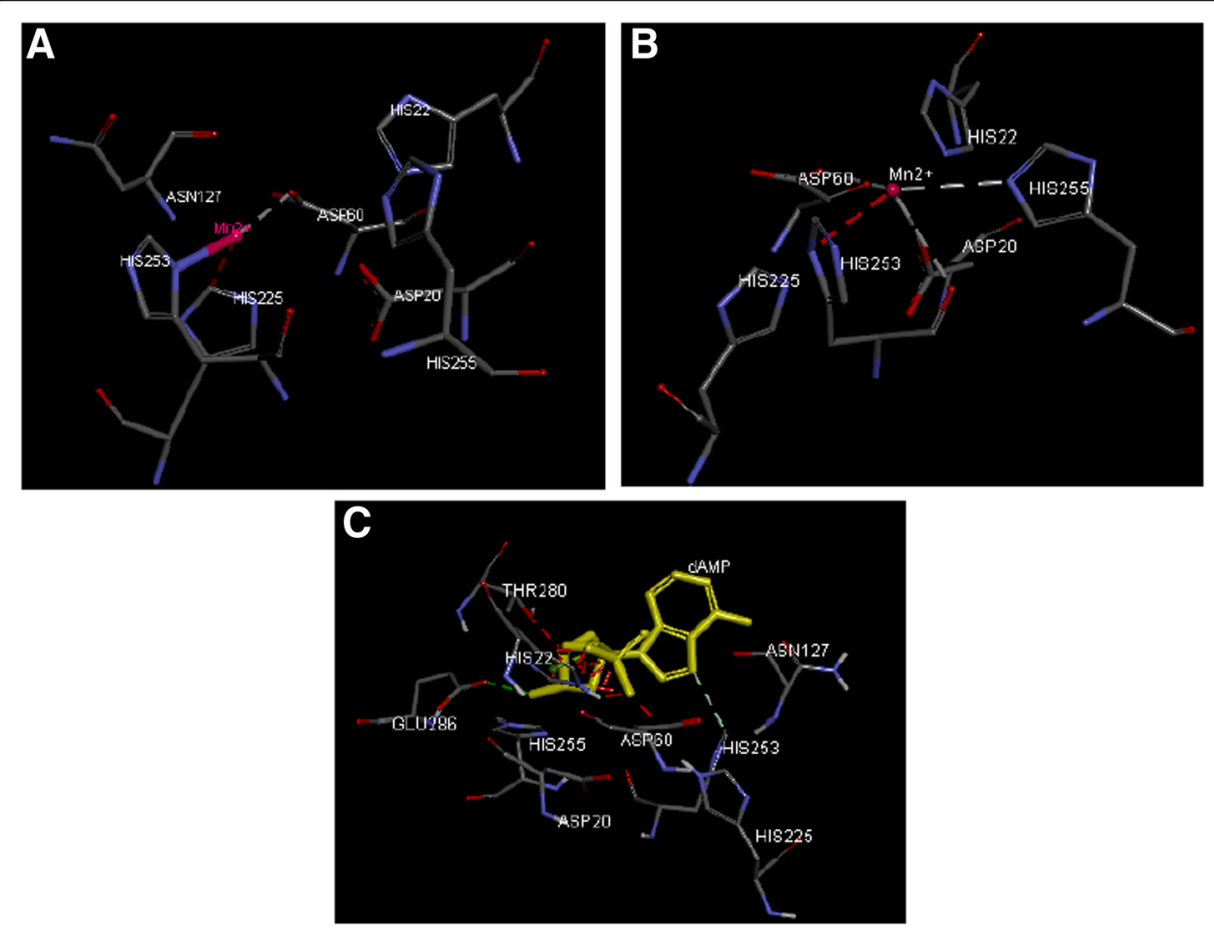

Fig. 4 The 3D structure of DnMRE11 showing its binding site. The proposed binding modes of $\mathrm{Mn} 2+$ and dAMP molecules are shown in stick format and noncarbon atoms are colored by atom type. Critical residues for binding of the first $\mathrm{Mn} 2+(\mathbf{a})$, second $\mathrm{Mn} 2+(\mathbf{b})$ and $\mathrm{dAMP}(\mathbf{c})$ are shown in this figure. The pink balls corresponding to the Mn 2+ ions and dAMP molecule are colored in yellow. Hydrogen bonds are shown in this figure with dotted lines 
residues that interact with metal coordinating residues, and all these residues are conserved in eukaryotes.

When we compared with the HmsMRE11sequence, we found the residues Arg80, Asp86, Asn116, and Pro120 of DnMRE11 that contribute to Nbs1 binding [29] are also conserved (Additional file 3), thus these residues may be involved in binding Nbs 1 by DnMRE11. The accessible surface areas in the amino acid sequence of DnMRE11 were predicted using the VADAR servers for model prediction (Additional file 5). We have predicted the ASA of each amino acid in the sequence along with the fractional residual volume available for the amino acids in the main as well as in the side chains. The quality of the model with respect to the stereo packing and 3D profile quality were also predicted using the VADAR server. The area accessible to water molecules on the protein structure is said to be accessible surface area (ASA), which was measured in square angstroms or as fractional ASA ranging from 0 to 1.

Hydrophilic residues occupied a large fraction of ASA with hydrophobic residues forming only a small fraction (Additional file 5). ASA values both for the whole structure and side chains were predicted using VADAR. The majority of residues in DnMRE11 have ASA scores less than 0.8 , indicating tight folding the generally leaves residues inaccessible to water molecules. We estimated the accessible surface areas of all residues of the active site and the Nbs1 binding site that are involved in the DnMRE11 3D structure using the VADAR server.

The ASA scores of the active site (Mn 2+ binding sites) were near zero (Asp $20: 0,01$; Asp60 : 0.03; His225: 0; His253 : 0.22; His255 :0,17) indicating the residues are not accessible to water molecules and confirmed that domain II (the capping domain) hides the active site. However, Nbs binding sites are exposed to surface (ASA scores $>0,8$ ).

\section{The plant MRE11 gene family}

We selected 50 genes in 41 taxa that appear to belong to the MRE11 family. The distribution of MRE11 genes among the various species is shown in Table 2.

Phylogenetic analysis was performed on the multiply aligned plant MRE11 proteins sequences, by the protein-maximum likelihood, using the Saccharomyces cerevisiae MRE11 SacMRE11 out as an outgroup (Additional file 6). This analysis revealed the presence of two major clusters corresponding to separate MRE11 gene subfamilies of monocots and eudicots species. Cluster 1 (blue) contains MRE11 sequences identified in the Liliopsida (monocots) group. Cluster 2 (red) contains MRE11 proteins from eudicots. A subcluster contained MRE11 sequences that were found in stem eudicotyledons (grey). We found that TspMRE11 and MicMRE11 (Chlorophyta, light blue), PhpMRE11 (Embryophyte, Green) and AmtMRE11 (Magnoliophyta, yellow) are separated from monocot and eudicot proteins. We found many duplication events in this tree. In cluster I, two duplication events were found between the two paralogous MRE11 proteins of the species Phoenix dactylifera DnMRE11 (Deglet nour variety), KhMRE11 (Khalas variety) and between the MRE11 paralogs of the species Musa acuminata (MuaMRE11X1, MuaMRE11X2). Two sequences, SeiMRE11X1and SeiMRE11- like, were identified which cluste red closely together within cluster 1 , suggesting that these paralogues were generated by a lineage specific duplication event. Other duplication events were unresolved as in cluster II, Cicer arietinum (CumMRE11X1, CumMRE11X2) and 
Table $\mathbf{2}$ List of accession numbers and list of taxa of plants used in this study

\begin{tabular}{|c|c|c|c|}
\hline Viridiplantae & & Ncbi accession code & Uniprot accession code \\
\hline \multicolumn{4}{|l|}{ Chlorophyta } \\
\hline Tetraselmis sp. GSL018 & TspMRE11 & JAC79210.1 & \\
\hline Micromonas sp. RCC299 & MicMRE11 & & C1ECB4 \\
\hline \multicolumn{4}{|l|}{ Embryophyta } \\
\hline Physcomitrella patens & PhpMRE11 & & G4XIR1 \\
\hline \multicolumn{4}{|l|}{ Magnoliophyta } \\
\hline Amborella trichopoda & AmtMRE11 & & W1P0X4 \\
\hline \multicolumn{4}{|l|}{ Stem eudicotyledons } \\
\hline Nelumbo nucifera & $\begin{array}{l}\text { NenMRE11X1 } \\
\text { NenMRE11X2 }\end{array}$ & $\begin{array}{l}\text { XP_010277930.1, } \\
\text { XP_010277931.1 }\end{array}$ & \\
\hline \multicolumn{4}{|l|}{ Eudicotyledons } \\
\hline Genlisea aurea & GeaMRE11 & & S8C042 \\
\hline Nicotiana tomentosiformis & NitMRE11X2 & XP_009613836.1 & \\
\hline Nicotiana sylvestris & NisMRE11X2 & XP_009787661.1 & \\
\hline Solanum tuberosum & SotMRE11like & XP_006341147.1 & \\
\hline Solanum lycopersicum & SolMRE11 & XP_004246548.2 & \\
\hline Vitis vinifera & VivMRE11 & XP_002281726.1 & \\
\hline Morus notabilis & MonMRE11 & EXB89636.1 & \\
\hline Prunus mume & PrmMRE11 & XP_008226714.1 & \\
\hline Malus domestica & MadMRE11X2 & XP_008366397.1 & \\
\hline Fragaria vesca subsp. vesca & FrvMRE11like & XP_004294486.1 & \\
\hline Populus trichocarpa & PotMRE11 & XP_006370340.1 & \\
\hline Ricinus communis & RicMRE11 & & B9SIEO \\
\hline Cucumis sativus & CusMRE11like & XP_004154884.1 & \\
\hline Cucumis melo & $\begin{array}{l}\text { CUMMRE11X1, } \\
\text { CumMRE11X2 }\end{array}$ & $\begin{array}{l}\text { XP_008454628.1 } \\
\text { XP_008454629.1 }\end{array}$ & \\
\hline Medicago truncatula & MetMRE11 & KEH38818.1 & \\
\hline Cicer arietinum & $\begin{array}{l}\text { CiaMRE11likeX2, } \\
\text { CiaMRE11X1 }\end{array}$ & $\begin{array}{l}\text { XP_004487655.1 } \\
\text { XP_004487654.1 }\end{array}$ & \\
\hline Glycine max & GImMRE1 1like & XP_003539581.1 & \\
\hline Eucalyptus grandis & EugMRE11 & XP_010060498.1 & \\
\hline Citrus clementina & CiCMRE11 & & V4URY3 \\
\hline Citrus sinensis & CisMRE11like & XP_006464669.1 & \\
\hline Eutrema salsugineum & EusMRE11 & & V4LH60 \\
\hline Brassica rapa & BrrMRE11 & XP_009119960.1 & \\
\hline Capsella rubella & CarMRE11 & & ROEVY8 \\
\hline Arabidopsis thaliana & ArtMRE11 & AED96476.1 & \\
\hline \multicolumn{4}{|l|}{ Liliopsida } \\
\hline $\begin{array}{l}\text { Musa acuminata subsp. } \\
\text { Malaccensis }\end{array}$ & $\begin{array}{l}\text { MuaMRE11X1 } \\
\text { MuaMRE11X2 }\end{array}$ & $\begin{array}{l}\text { XP_009412711.1 } \\
\text { XP_009412712.1 }\end{array}$ & \\
\hline $\begin{array}{l}\text { Phoenix dactylifera } \vee \text { Deglet } \\
\text { nour }\end{array}$ & DnMRE11 & & \\
\hline Phoenix dactylifera $\vee$ Khalas & KhMRE11 & XP_008803852.1 & \\
\hline Zea mays & ZemMRE11 & NP_001151499.1 & \\
\hline Sorghum bicolor & SobMRE11 & & C5YGR2 \\
\hline
\end{tabular}


Table 2 List of accession numbers and list of taxa of plants used in this study (Continued)

\begin{tabular}{llll}
\hline Setaria italica & SeiMRE11X1, & XP_004976962.1 & \\
SeiMRE11like & XP_004974387.1 & \\
Oryza brachyantha & OrbMRE11like & XP_006652883.1 & \\
Oryza sativa Group Japonica & OrsMRE11 & & Q7XQR9 \\
Brachypodium Distachyon & BrdMRE11like, & XP_003571409.1 & \\
& BadMRE11 & XP_010240488.1 & \\
Aegilops tauschii & AetMRE11 & EMT05178.1 & Q4GX62 \\
Triticum turgidum & TrtMRE11 & & W5B7S5 \\
Triticum aestivum & TraMRE11 & & F2DMY1 \\
Hordeum vulgare & HovMRE11 & & \\
\hline
\end{tabular}

Cucumis melo (CumMRE11X1, CumMRE11X2). Relationships among MRE11 genes from monocots, stem eudicots, core eudicots, Chlorophyta, Embryophyte and Magnoliophyta were inferred from the conserved nuclease, capping and DNA binding regions. The ConSurf server was used to extract information about important residues, which are of functional value. This server provides evolutionary conservation scores for residues, which could be correlated with biological function. In our case, the ConSurf server predicted that residues of the active site and Nbs binding site have high conservation scores (dark pink in Table 3). Among these, D20 and P120 were found to have high scores, indicating evolutionary conservation and hence important functional roles (Additional file 7 and Table 3). Approximately $85 \%$ of the residues are conserved among the nuclease and capping domains shown in Additional files 3 and 7.

Most of the variation within this region occurs in the capping domain.

\section{Conclusion}

In silico analysis of DnMRE11 was conducted by motif analysis and phylogenetic tree construction using PhyML. The ConSurf server predicted that residues of the active site and Nbs binding site have high conservation scores. A model structure of DnMRE11 was constructed using Swiss-Model server using homology-based modelling

Table 3 The table details the residue variety in \% for each position in the query sequence. Each column shows the \% for that amino-acid, found in position ('pos') in the MSA

\begin{tabular}{lll}
\hline Amino acid position of actif site & Pourcentage of concervation & Consurf grade \\
\hline D20 & 100 & 9 \\
H22 & 98 & 9 \\
N127 & 98 & 9 \\
H225 & 98 & 9 \\
H253 & 98 & 9 \\
H255 & 98 & 9 \\
Amino acid position of Nbs binding site & & \\
R80 & 94 & 9 \\
D86 & 98 & 9 \\
N116 & 98 & 9 \\
P120 & 100 & 9 \\
\hline
\end{tabular}


and validated with ProSA, QMEAN servers and Ramachandran plot analysis, which suggested the predicted model to be satisfactory. Further validation studies were conducted by $\mathrm{COACH}$ analysis for active site ligand prediction, and revealed the presence of six ligands binding sites and two ligands (2 Mn2+ and dAMP).

\title{
Additional files
}

\author{
Additional file 1: Results of BLASTX and annotation of a potential candidate cDNA of the DnMRE11. \\ (PNG $148 \mathrm{~kb}$ ) \\ Additional file 2: Structure guided sequence alignment of DnMRE11 with Schizosaccharomyces pombe \\ (4fbk.1.A) MRE11. (PNG 103 kb)
}

Additional file 3: The alignment between DnMRE11, Aeropyrum pernix K1 (AepMRE11, archaea), Homo sapiens (HmsMRE11, animals), Kocuria sp. UCD OTCP (KocMRE11, bacteria), Saccharomyces cerevisiae (SacMRE11, fungi) and Galdieria sulphuraria (GasMRE11, protista). Conserved residues are red. The five conserved phosphodiesterase motifs, which form the nuclease active site, are showns in this figure with roman numbers. (PNG $770 \mathrm{~kb}$ )

Additional file 4: Prediction of the active site location of DnMRE11 by the DEPTH server. (PNG 133 kb) Additional file 5: Fractional accessible surface area of DnMRE11. (PNG $94 \mathrm{~kb}$ )

Additional file 6: Phylogenetic maximum likelihood tree showing the evolutionary relationships among MRE11 proteins from 50 plant species. Tree was created using the MUSCLE alignment tool and EvolView software. Bootstrap values are indicated in tree adjacent to the relevant branches. Eudicotyledons are highlighted in pink, Chlorophyta in light blue, Liliopsida (monocots) in blue, Embryophyta in green, Magnoliophyta in yellow, and stem eudicotyledons in grey. (PNG $186 \mathrm{~kb}$ )

Additional file 7: Results of ConSurf analysis mapped onto the MRE11 structure (residues 1-404) using the maximum likelihood method. Shown on the left is the space filling view of the protein; right, the opposite side following a $180^{\circ}$ rotation about the $y$ axis. Conserved residues are darkest pink, variable residues are cyan, and others are white. (PNG $241 \mathrm{~kb}$ )

Competing interests

The authors declare that they have no competing interests.

Authors' contributions

All authors read and approved the final manuscript.

Acknowledgments

This work was supported by grants from the Tunisian minister of Higher Education and Scientific Research.

Disclosure

The authors report no disclosures or financial support.

Author details

${ }^{1}$ Laboratory of plant biotechnology, Faculty of sciences of Sfax, University of Sfax, Sfax, Tunisia. ${ }^{2}$ Leibniz Institute of Plant Biochemistry, Halle, Germany. ${ }^{3}$ High Institute of Applied Biology of Medenine, Medenine, Tunisia.

Received: 22 April 2015 Accepted: 26 August 2015

Published online: 05 November 2015

References

1. Duvall MR, Clegg MT, Chase MW, Clark WD, Kress JW, Zimmer EA, et al. Phylogenetic hypotheses for the Monocotyledons constructed from rbcL sequence data. Ann Mo Bot Gard. 1993;80:607-19.

2. Stewart GS, Maser RS, Stankovic T, Bressan DA, Kaplan MI, Jaspers NG, et al. The DNA double-strand break repair gene hMRE11 is mutated in individuals with an ataxia-telangiectasia-like disorder. Cell. 1999;99:577-87.

3. Michel B, Ehrlich SD, Uzest M. DNA double strand breaks caused by replication arrest. EMBO J. 1997;16:430-8.

4. Leach DRF. Genetic recombination. Oxford: Blackwell Science; 1996.

5. Amours D, Jackson SP. The MRE11 complex: at the crossroads of dna repair and checkpoint signalling. Nat Rev Mol Cell Biol. 2002;3:317-27.

6. Stracker TH, Petrini JH. The MRE11 complex: starting from the ends. Nat Rev Mol Cell Biol. 2011;12:90-103.

7. Mimitou EP, Symington LS. DNA end resection: many nucleases make light work. DNA Repair (Amst). 2009;8:983-95.

8. Trujillo KM, Roh DH, Chen L, Van Komen S, Tomkinson A, Sung P. Yeast xrs2 binds DNA and helps target rad50 and MRE11 to DNA ends. J Biol Chem. 2003;278:48957-64.

9. Desai Mehta A, Cerosaletti KM, Concannon P. Distinct functional domains of nibrin mediate MRE11 binding, focus formation, and nuclear localization. Mol Cell Biol. 2001;21:2184-91.

10. Lim HS, Kim JS, Park YB, Gwon GH, Cho Y. Crystal structure of the MRE11, Rad50, ATPgS complex: understanding the interplay between MRE11 and Rad50. Genes Dev. 2011;25:1091-104.

11. Williams RS, Moncalian G, Williams JS, Yamada Y, Limbo O, Shin DS, et al. MRE11 dimers coordinate DNA end bridging and nuclease processing in double strand break repair. Cell. 2008;135:97-109. 
12. Falck J, Coates J, Jackson SP. Conserved modes of recruitment of ATM, ATR and DNA, PKcs to sites of DNA damage. Nature. 2005;434:605-11.

13. Hartung F, Puchta H. Isolation of the complete CDNA of the MRE11 homologue of Arabidopsis (accession no AJ243822) indicates conservation of DNA recombination mechanisms between plants and other eukaryotes. Plant Physiol. 1999;121:312.

14. Stracker TH, Theunissen JW, Morales M, Petrini JH. The MRE11 complex and the metabolism of chromosome breaks: the importance of communicating and holding things together. DNA RepairAmst. 2004;3:845-54.

15. Jager M, van Noort J, van Gent DC, Dekker C, Kanaar R. Human Rad50/MRE11 is a flexible complex that can tether DNA ends. Mol Cell. 2001;8:1129-35.

16. Ajimura M, Leem SH, Ogawa H. Identification of new genes required for meiotic recombination in Saccharomyces cerevisiae. Genetics. 1993;133:51-66.

17. Zhao SY, Wu YR, Xia GM. Introduction of a simple and effective method for plant total RNA isolation. Yi Chuan. 2002:24:337-8.

18. Huang X, Madan A. CAP3: a DNA sequence assembly program. Genome Res. 1999;9:868-77.

19. Altschul SF, Madden TL, Schaffer AA, Zhang J, Zhang Z, Miller W, et al. Gapped BLAST and PSI, BLAST: a new generation of protein database search programs. Nucleic Acids Res. 1997;25:3389-402.

20. Conesa A, Götz S. "Blast2GO: a comprehensive suite for functional analysis in plant genomics". Int J Plant Genomics. 2008;1-13. doi: 10.1155/2008/619832.

21. Eddy SR. A new generation of homology search tools based on probabilistic inference. Genome Inform. 2009;23:205-11.

22. Edgar RC. MUSCLE: multiple sequence alignment with high accuracy and high throughput. Nucleic Acids Res. 2004:32:1792-7.

23. Guindon S, Gascuel O. A simple, fast, and accurate algorithm to estimate large phylogenies by maximum likelihood. Syst Biol. 2003;52:696-704.

24. Zhang H, Gao S, Lercher MJ, Hu S, Chen WH. EvolView, an online tool for visualizing, annotating and managing phylogenetic trees. Nucleic Acids Res. 2012;40:W569-72

25. Benkert P, Künzli M, Schwede T. "QMEAN server for protein model quality estimation". Nucleic Acids Res. 2009:37(Web Server issue):W510-4

26. Wiederstein $M$, Sippl MJ. ProSA-web: interactive web service for the recognition of errors in three-dimensional structures of proteins. Nucl Acids Res. 2007:3:407-10.

27. Yang J, Roy A, Zhang Y. Protein-ligand binding site recognition using complementary binding, specific substructure comparison and sequence profile alignment. Bioinformatics. 2013;29:2588-95

28. Aravind L, Walker DR, Koonin EV. Conserved domains in DNA repair proteins and evolution of repair systems. Nucleic Acids Res. 1999;27:1223-42.

29. Christian BS, Katja L, Ilaria G, Britta C, Heidi F, Florian S, et al. Structure of MRE11-Nbs1 complex yields insights into ataxia, telangiectasia-like disease mutations and DNA damage signaling. Nat Struct Mol Biol. 2012;19:693-700.

30. Park YB, Chae J, Kim YC, Cho Y. Crystal structure of human MRE11: understanding tumorigenic mutations. Structure. 2011;19:1591-602.

31. Griffith JP, Kim JL, Kim EE, Sintchak MD, Thomson JA, Fitzgibbon MJ, et al. X ray structure of calcineurin inhibited by the immunophilin, immunosuppressant FKBP12-FK506 complex. Cell. 1995;82:507-22.

32. Wass MN, Kelley LA, Sternberg MJ. 3DLigandSite: predicting ligand, binding sites using similar structures. Nucleic Acids Res. 2010;38(Web Server issue):W469-73.

33. Hopfner KP, Karcher A, Craig L, Woo T, Carney JP, Tainer JA. Structural biochemistry and interaction architecture of the DNA double strand break repair MRE11 nuclease and Rad50, ATPase. Cell. 2001:105:473-85.

\section{Submit your next manuscript to BioMed Central and take full advantage of:}

- Convenient online submission

- Thorough peer review

- No space constraints or color figure charges

- Immediate publication on acceptance

- Inclusion in PubMed, CAS, Scopus and Google Scholar

- Research which is freely available for redistribution

Submit your manuscript at www.biomedcentral.com/submit 